\title{
Elective neurosurgical humanitarian care in a deployed setting
}

\author{
Jonathan A. Forbes, MD \\ Department of Neurological Surgery, University of Cincinnati College of Medicine, Cincinnati, Ohio
}

OBJECTIVE Active-duty neurosurgical coverage has been provided at Bagram Air Force Base in Afghanistan since 2007. Early operative logs were reflective of a large number of surgical procedures performed to treat battlefield injuries. However, with maturation of the war effort, the number of operations for battlefield injuries has decreased with time. Consequently, procedures performed for elective neurosurgical humanitarian care $(\mathrm{NHC})$ increased in number and complexity prior to closure of the Korean Hospital in 2015, which resulted in effective termination of NHC at Bagram. Monthly neurosurgical caseloads for deployed personnel have dropped precipitously since this time, renewing a debate as to whether the benefits of providing elective NHC in Afghanistan outweigh the costs of such a strategy. To date, there is a paucity of information in the literature discussing the overall context of such a determination.

METHODS The author retrospectively reviewed his personal database of all patients who underwent neurosurgical procedures at Bagram during his deployment there from April 17 to October 29, 2014. Standardized clinical parameters had been recorded in the ABNS NeuroLog system. All cases of nonelective surgical care for battlefield injuries were identified and excluded. Records of all other procedures, which represented elective NHC delivered during this period, were accessed to extract salient clinical and radiological data.

RESULTS During the 6 -month deployment, 49 patients ( 29 male and 20 female, age range 18 months to 63 years) were treated by the author in elective NHC. Procedures were performed for spinal degenerative disease $(n=28)$, cranial tumors $(n=11)$, pediatric conditions $(n=6)$, Pott's disease $(n=2)$, peripheral nerve impingement $(n=1)$, and adult hydrocephalus $(n=1)$. The duration of follow-up ranged from 3 to 23 weeks. Complications referable to surgery included asymptomatic, unilateral lumbar screw fracture detected 3 months postoperatively and treated with revision of hardware $(n=1)$; wound infection requiring cranial flap explantation and staged cranioplasty $(n=1)$; and unanticipated return to the operating room for resection of residual tumor in a patient with a solitary metastatic lesion involving the mesial temporal lobe/ambient cistern $(n=1)$. There were no instances of postoperative neurological decline.

CONCLUSIONS Elective NHC can be safely and effectively implemented in the deployed setting. Benefits of a military strategy that supports humanitarian care include strengthening of the bond between the US/Afghan military communities and the local civilian population as well as maintenance of skills of the neurosurgical team during the sometimes-lengthy intervals between cases in which emergent neurosurgical care is provided for treatment of battlefield injuries.

https://thejns.org/doi/abs/10.3171/2018.8.FOCUS18366

KEYWORDS military; neurosurgery; deployment; humanitarian care; counterinsurgency platform; Craig Joint Theater Hospital

$\mathrm{R}$ ECENT articles have highlighted difficulties associated with the low annual caseloads reported by military surgeons..$^{10}$ This phenomenon is especially evident in the contemporary deployed setting, where it is not uncommon for military surgeons to perform less than 1 surgical case a month. ${ }^{3,9}$ Faced with the prospect of declining caseloads for extended periods, the military surgical community has expressed concern about potential erosion of skills.
Since 2007, no less than 1 active-duty neurosurgeon has provided continuous neurosurgical coverage at Bagram Air Force Base (AFB) in Afghanistan. Operative logs in earlier phases of coverage were reflective of a large number of surgical procedures performed to treat battlefield injuries. ${ }^{1,7}$ With maturation of the war effort and the Afghan military medical network, the number of operations for battlefield injuries has decreased. In response to this trend, cases of elective neurosurgical humanitarian care (NHC)

ABBREVIATIONS ACA = anterior cerebral artery; $\mathrm{ACDF}=$ anterior cervical discectomy and fusion; $\mathrm{AFB}=\mathrm{Air}$ Force Base; $\mathrm{CJTH}=\mathrm{Craig}$ Joint $\mathrm{Theater}$ Hospital; $\mathrm{HC}=$ humanitarian care; $\mathrm{MCA}=$ middle cerebral artery; $\mathrm{NHC}=$ neurosurgical humanitarian care; $\mathrm{OR}=$ operating room; $\mathrm{SSS}=$ superior sagittal sinus.

SUBMITTED July 22, 2018. ACCEPTED August 7, 2018.

INCLUDE WHEN CITING DOI: 10.3171/2018.8.FOCUS18366. 
increased in number and complexity prior to closure of the Korean Hospital in 2015, which resulted in a marked decrease in NHC at Bagram. Since closure of the Korean gate of entry, monthly neurosurgical caseloads have dropped precipitously-renewing a debate as to whether the benefits of providing elective NHC in Afghanistan outweigh the costs of such a strategy. To date, there is a paucity of information in the literature discussing the overall context of such a determination. In this article, patient characteristics and clinical outcomes of a consecutive series of cases in which patients were provided elective $\mathrm{NHC}$ by the author during his deployment are reported.

\section{Methods}

\section{Preoperative Data Collection}

The author retrospectively reviewed his personal database of all patients who underwent neurosurgical procedures at Craig Joint Theater Hospital (CJTH) at Bagram AFB during time spent deployed in 2014. A summary of all clinical details-including initial consultations, operative notes, postoperative complications, and postoperative visits - had been recorded in the ABNS (American Board of Neurological Surgery) NeuroLog system. Duplicate copies of these records were accessed to extract relevant information. All cases of nonelective surgical care for battlefield injuries involving NATO troops, non-NATO allied troops, enemy combatants, and local Afghan civilians were identified and excluded. Records of all other procedures, which represented elective NHC delivered during this period, were accessed in a de-identified manner to extract standardized clinical and radiological data.

\section{Intraoperative and Postoperative Data Collection}

Relevant intra- and postoperative data (e.g., surgical approach, intraoperative complications, postoperative neurological deficit, postoperative complications, and radiological and clinical follow-up) were assessed following surgery. Extent of tumor resection was assessed postoperatively using CT and MRI when available. Histopathologic specimens and nonroutine (e.g., endocrinologic) samples for laboratory testing were sent to Landstuhl Regional Medical Center in Germany for analysis. The results of these studies could be accessed via the Armed Forces Health Longitudinal Technology Application (AHLTA) medical records system. Full neurological examinations were performed on a daily basis in the inpatient setting following surgery and during follow-up in the outpatient setting.

\section{Results}

During the period from April 17 to October 29, 2014, 49 patients ( 29 male and 20 female) were treated by the author at CJTH in elective NHC. At the time of surgery, the ages of the patients ranged from 18 months to 63 years old. Procedures were performed for spinal degenerative disease $(n=28)$, resection of cranial tumors $(n=11)$, pediatric conditions $(n=6)$, Pott's disease $(n=2)$, peripheral nerve impingement $(\mathrm{n}=1)$, and treatment of hydrocephalus $(\mathrm{n}=$ 1). Follow-up with notes available for review ranged from 3 to 23 weeks. Select patients underwent lengthier follow- up with antecedent active-duty, deployed neurosurgeons; an Afghan neurosurgical trainee who was involved in select cases; and/or personal communication via emailhowever, data from these interactions were not formally reviewed for the purposes of this study. Complications referable to surgery included wound infection requiring cranial flap explantation and staged cranioplasty $(n=1)$, asymptomatic lumbar screw fracture detected 3 months following surgery treated with revision of hardware $(\mathrm{n}=$ 1), and unanticipated return to the operating room (OR) for resection of residual tumor in a patient with a solitary metastatic lesion involving the mesial temporal lobe/ambient cistern $(n=1)$. There were no instances of postoperative neurological decline.

A summary of elective NHC procedures performed can be found in Table 1 . While a variety of spinal disorders could be treated at CJTH, some institutional limitations merit additional mention. CJTH was equipped with pedicle screws, lateral mass screws, and anterior cervical plates. However, there were no expandable or stackable cages, no options for cadaveric allograft, no anterior thoracolumbar locking plate/screw constructs available, and no electrophysiological monitoring. When structural reconstruction of the anterior column was required, it was common to harvest iliac crest autograft for ACDF (anterior cervical discectomy and fusion) and/or ALIF (anterior lumbar interbody fusion) procedures and fibular autograft to reconstruct larger defects that followed corpectomies. Preference was given to lumbar decompression alone when possible. In patients requiring fusion across L5-S1 (e.g., for symptomatic L5-S1 spondylolisthesis), circumferential fusion was routinely performed to maximize postoperative rates of arthrodesis. The use of iliac crest autograft to fashion TLIF (transforaminal lumbar interbody fusion) grafts was avoided, as previous surgeons at CJTH had noted a tendency for post-implantation graft retropulsion using this method. Of the cases listed in Table 1, 5 involved revisions following previous surgeries performed by outside surgeons. One patient in the series, who underwent a posterior lumbar decompression and fusion, was found to have an asymptomatic, unilateral L5 screw fracture on routine plain radiographs obtained 3 months following surgery. Although his symptoms had resolved following the initial surgery, the decision was made to return to the OR for hardware revision to minimize the possibility of long-term complications.

Institutional limitations regarding treatment of cranial pathology included the inability to obtain MRI studies at $\mathrm{CJTH}$, lack of neuronavigation and high-quality microscopy (Fig. 1), and inability to perform cortical mapping. Additionally, hospital leadership during this period requested that all cases longer than 4 hours in duration be staged to maximize operative capabilities in the event of incoming mass casualty. With this policy in mind, in 2 cranial cases (one involving an intraventricular neoplasm and the other a large petroclival meningioma) the operations were completed in 2 stages. In a third case, in which a right frontotemporal craniotomy was performed for resection of a metastatic lesion involving the mesial temporal lobe with extension into the ambient cistern, postoperative imaging demonstrated a small amount of residual 
TABLE 1. Summary of elective NHC procedures performed at Bagram AFB from April 17 to October 29, 2014

\begin{tabular}{|c|c|c|}
\hline Type of Surgery & $\begin{array}{c}\text { No. of } \\
\text { Patients }\end{array}$ & Additional Details \\
\hline \multicolumn{3}{|l|}{ Spine } \\
\hline Degenerative, cervical & 4 & 3 ACDF; 1 PCLF \\
\hline Degenerative, lumbar & 24 & $\begin{array}{c}12 \text { decompression alone; } 12 \\
\text { decompression + fusion }\end{array}$ \\
\hline Infectious, Pott's disease & 2 & $\begin{array}{c}2 \text { anterior multi-level corpec- } \\
\text { tomy + posterior fusion }\end{array}$ \\
\hline \multicolumn{3}{|l|}{ Cranial } \\
\hline $\begin{array}{l}\text { Meningioma (PS: 1; CO: } \\
\text { 1; SO: 1; PC: 1) }\end{array}$ & 4 & GTR: 2; NTR: 1; STR: 1 \\
\hline Glioma (IV: 1; LF: 1; LT: 1) & 3 & GTR: 3 \\
\hline $\begin{array}{l}\text { Pituitary adenoma (GH: } \\
\text { 1; NF: 2) }\end{array}$ & 3 & GTR: 2; NTR: 1 \\
\hline Metastasis (RMT: 1) & 1 & GTR: $1^{*}$ \\
\hline \multicolumn{3}{|l|}{ Pediatrics } \\
\hline Encephalocele & 2 & 2 occipital \\
\hline $\begin{array}{l}\text { Hydrocephalus (AC/QC: } \\
\text { 1; AS: 1) }\end{array}$ & 2 & $\begin{array}{l}\text { Treatment using endoscopic } \\
\text { fenestration }\end{array}$ \\
\hline $\begin{array}{l}\text { Growing skull fracture } \\
\text { (FTP: 1) }\end{array}$ & 1 & - \\
\hline $\begin{array}{l}\text { Infection secondary to } \\
\text { penetrating injury }(F: 1)\end{array}$ & 1 & - \\
\hline \multicolumn{3}{|l|}{ Miscellaneous } \\
\hline $\begin{array}{l}\text { Communicating hydro- } \\
\text { cephalus, adult }\end{array}$ & 1 & Treated w/ VP shunt \\
\hline $\begin{array}{l}\text { Peripheral nerve impinge- } \\
\text { ment }\end{array}$ & 1 & Treated $\mathrm{w} / \mathrm{CT}$ release \\
\hline
\end{tabular}

$\mathrm{AC}=$ ambient cistern; $\mathrm{ACDF}=$ anterior cervical discectomy and fusion; $\mathrm{AS}=$ aqueductal stenosis; $\mathrm{CO}=$ convexity; $\mathrm{CT}=$ carpal tunnel; $\mathrm{F}=$ frontal; $\mathrm{FTP}=$ frontotemporoparietal; $\mathrm{GH}=$ growth hormone-secreting; $\mathrm{GTR}=$ gross-total resection; IV = intraventricular; $L F=$ left (dominant) frontal; $L T=$ left (dominant) temporal; NF = nonfunctioning; NTR = near-total resection; $P C=$ petroclival; $\mathrm{PCLF}=$ posterior cervical laminectomy and fusion; $\mathrm{PS}=$ parasagittal; $\mathrm{QC}=$ quadrigeminal cistern; RMT = right mesial temporal/ambient cistern; $\mathrm{SO}=$ spheno-orbital; STR = subtotal resection; VP = ventriculoperitoneal.

Spinal procedures are stratified by location, cranial procedures are stratified by pathology, and pediatric procedures are stratified by etiology.

* GTR achieved following unanticipated return to OR.

neoplasm; the decision was made to return to the OR to achieve gross-total resection. In another case of surgical morbidity, a patient who underwent resection of a sphenoorbital meningioma returned approximately 3 weeks following surgery with pus draining from the incision and required additional surgery for washout with cranial flap explantation. After receiving a prolonged course of antibiotic therapy, he returned to the OR for cranioplasty in a delayed manner and suffered no additional complications. Altogether, gross-total resection $(n=8)$ or near-total resection (defined as resection of $>95 \%$ of the tumor, $\mathrm{n}=$ 2) was achieved in 10 of 11 tumor cases $(91 \%)$. In the 2 cases of near-total resection, cavernous sinus invasion (n $=1)$ and superior sagittal sinus invasion $(\mathrm{n}=1)$ prevented gross-total resection. One massive petroclival meningio-

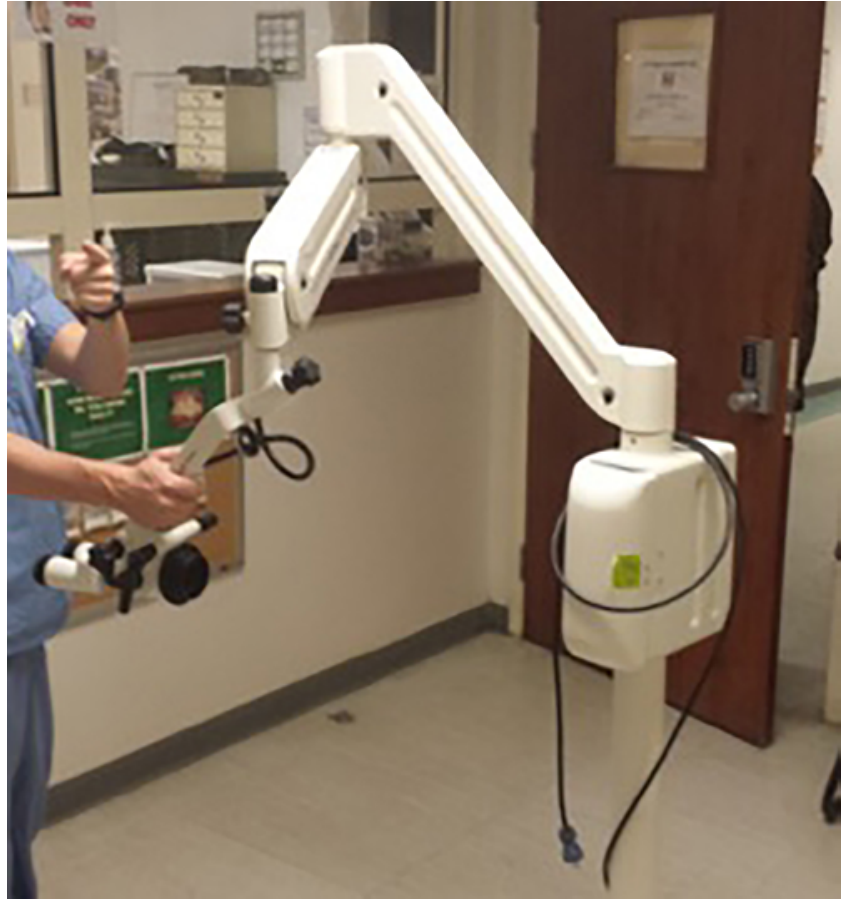

FIG. 1. Operative microscope at CJTH.

ma was treated with subtotal resection (resection of $<95 \%$ of the tumor), with marked improvement in balance noted postoperatively.

Institutional limitations regarding treatment of pediatric pathology included lack of access to a standardized ventriculoscope. However, a makeshift ventriculoscope could be constructed by tethering a $2.7-\mathrm{mm}$ endoscope to a 5-Fr pediatric feeding tube, which provided irrigation for neuroendoscopic visualization. In turn, it was possible to loosely couple this apparatus with micrograspers from a urology tray to permit intraventricular fenestration (used successfully to treat a quadrigeminal arachnoid cyst and perform an endoscopic third ventriculostomy for aqueductal stenosis). Of note, a fellowship-trained pediatric anesthesiologist was available to provide anesthesia for all pediatric procedures. In addition to the cases described above, 2 miscellaneous procedures were performed: 1 carpal tunnel release and 1 ventriculoperitoneal shunt insertion in an adult patient who presented with headaches and communicating hydrocephalus.

\section{Illustrative Cases \\ Case 1}

A 60-year-old man presented with severe weakness of the left side (leg $>$ arm) leading to progressive, marked difficulty with ambulation. On initial examination, strength was 3/5 in his left upper extremity, 2/5 in his proximal left lower extremity, and 1/5 in his distal left lower extremity. A CT scan of the head with contrast demonstrated a large right parasagittal tumor involving the posterior frontal region with mass effect on the motor cortex (Fig. 2A). Preoperative angiography was obtained using $\mathrm{C}$-arm fluoroscopy (Fig. 2B) and demonstrated residual flow through 


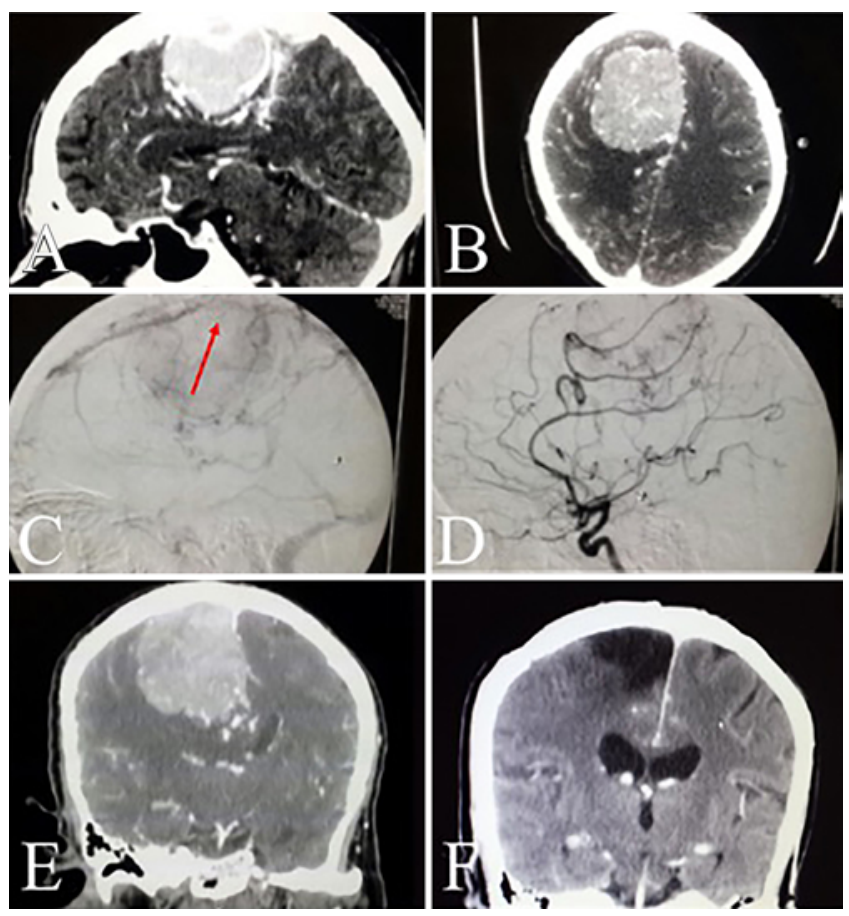

FIG. 2. A and B: Preoperative sagittal $(A)$ and axial $(B)$ postcontrast CT images demonstrating a large, parasagittal extra-axial neoplasm with mass effect on the right posterior frontal lobe. C: Right internal carotid angiogram, venous phase, lateral view. The mass effect of tumor narrows the SSS, but persistent flow through the SSS is present (red arrow). D: Right internal carotid angiogram, mid-arterial phase, lateral view providing excellent view of configuration of distal ACA circulation, which is intimately involved with the tumor capsule. E and F: Preoperative $(E)$ and postoperative $(F)$ coronal postcontrast CT images demonstrating resection of all tumor components exterior to the superior sagittal sinus. Near-total (>95\%) resection was achieved.

the superior sagittal sinus (SSS). Images from the arterial phase (Fig. 2C) provided an excellent view of the distal anterior cerebral distribution, which was noted to be intimately involved with the inferomedial tumor capsule. In the setting of progressive motor deficit, surgery was recommended. In the OR, the patient was positioned supine and his head was placed in Mayfield pin fixation. A linear incision in the coronal plane was fashioned. A craniotomy biased to the right, exposing the SSS, was turned. The tumor was internally debulked prior to circumdissection of the tumor capsule off the posterior inferior frontal artery branch of the distal anterior cerebral artery (ACA) circulation, which was preserved. Tumor resection continued to the superior sagittal sinus, where all tumor exterior to the SSS was disconnected and removed. Postoperative imaging demonstrated expected residual tumor in the SSS; otherwise, no residual tumor was present. Radiological findings were consistent with near-total (>95\%) resection. The patient noted considerable improvement in strength following surgery. At follow-up 3 months postoperatively, strength in his left upper extremity was graded at $4+/ 5$. Strength in his left lower extremity had improved to $4+/ 5$ proximally and 4-/5 distally. He was able to ambulate with minor difficulty at this time.
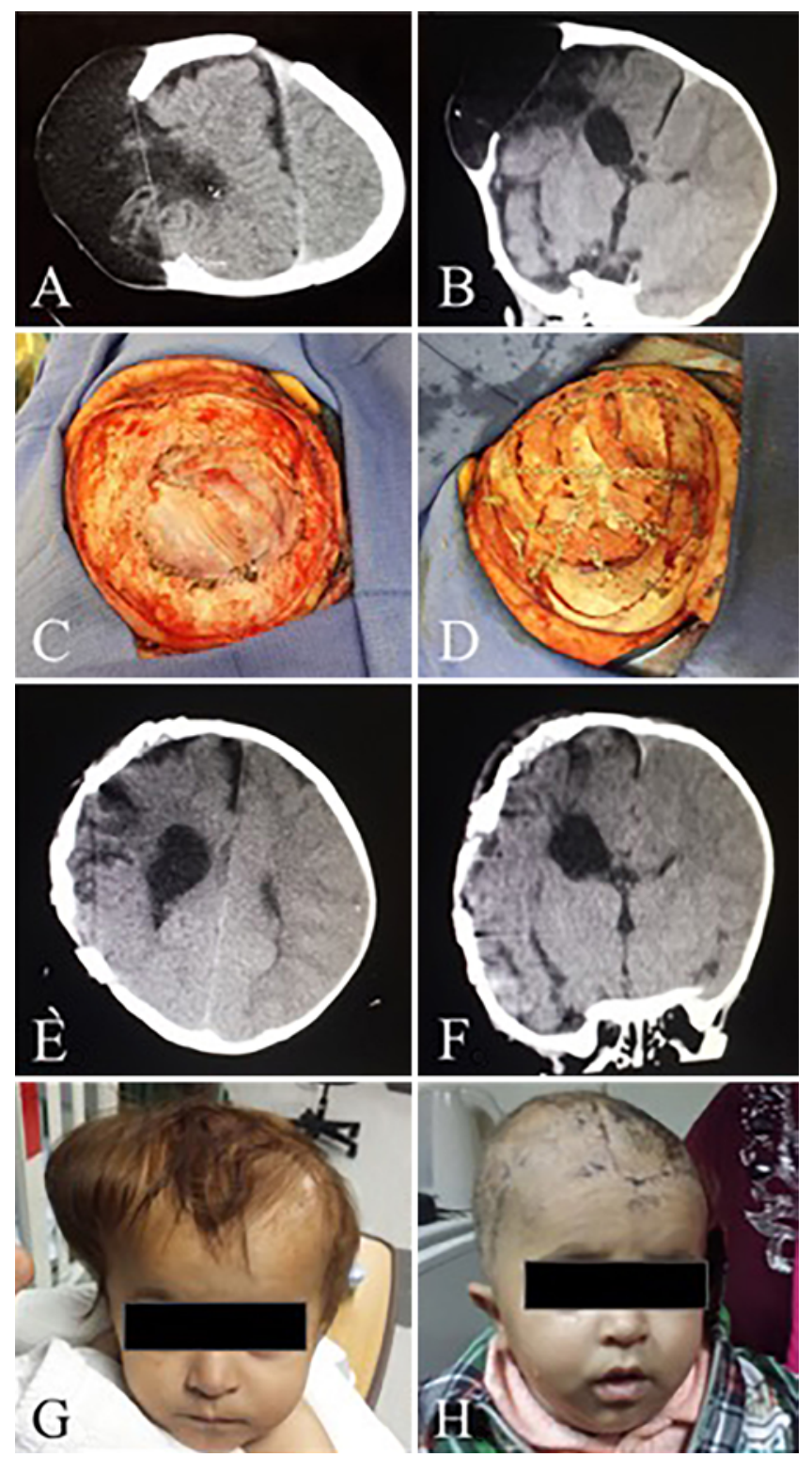

FIG. 3. A and B: Axial (A) and coronal (B) preoperative noncontrast CT of the head, which demonstrates findings consistent with a growing skull fracture. C and D: Intraoperative views following pericranial repair of dural defect $(C)$ and calvarial reconstruction using split-thickness calvarial autograft (D). E and F: Postoperative axial (E) and coronal (F) noncontrast CT images demonstrating calvarial reconstruction. $\mathbf{G}$ and $\mathrm{H}$ : Preoperative $(\mathrm{G})$ and postoperative $(\mathrm{H})$ photographs demonstrating favorable cosmesis following surgery.

\section{Case 2}

An 18-month-old male with a history of a severe head injury 6 months prior presented with an enlarging right frontoparietal pulsatile mass. A noncontrast CT scan of the head was performed, revealing findings consistent with a large, growing skull fracture (Fig. 3A and B). The decision was made to proceed with surgical repair. In the OR, the patient's head was placed on a horseshoe headrest. A curvilinear reverse question-mark incision beginning at the level of the zygoma was fashioned on the right. The 

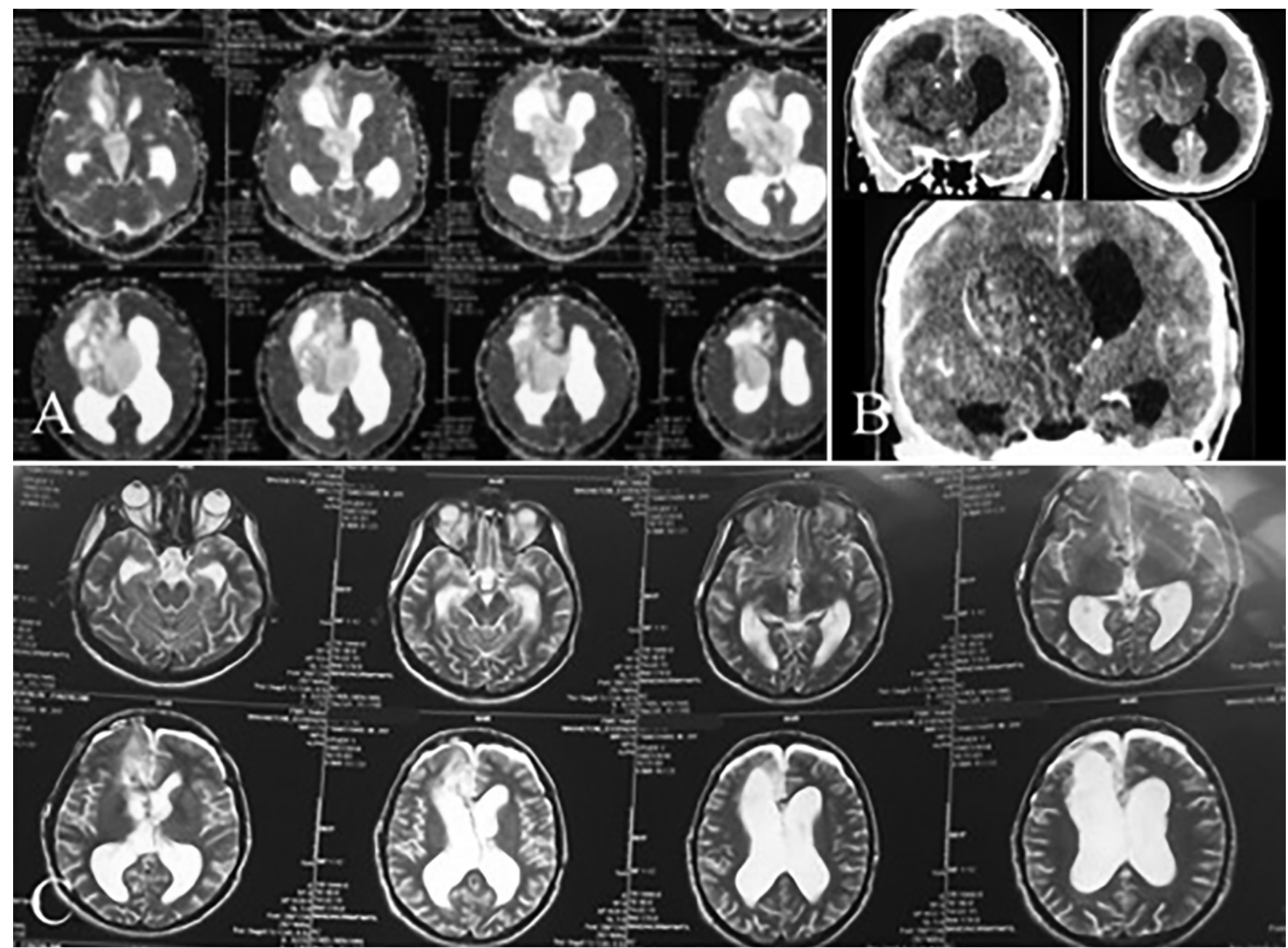

FIG. 4. A: Preoperative T2-weighted axial MR images demonstrating a large intraventricular neoplasm extending from the third ventricle into the right lateral ventricle with infiltration of overlying right frontal lobe. B: Postcontrast CT images obtained following evaluation at Bagram demonstrating interval enlargement of the neoplasm with progression in associated hydrocephalus. C: Postoperative MR image demonstrating gross-total resection of the neoplasm and interval improvement in the associated hydrocephalus.

scalp flap was turned in the subgaleal plane, and a large pericranial flap was harvested. Burr holes were placed, and a large frontotemporoparietal cranial flap was turned; the flap was subsequently split using a reciprocating saw and thin chisel to facilitate autologous coverage. The intact dura mater was exposed around the dural defect and dystrophic brain was dissected free from the dural laceration. The overlying cerebromeningeal cicatrix was removed with bipolar electrocautery and sharp dissection. The large dural defect was repaired with pericranial autograft (Fig. 3C). The large calvarial defect was repaired with split-thickness calvarial bone obtained from the craniotomy flap (Fig. 3D). A postoperative CT scan demonstrated adequate calvarial reconstruction (Fig. 3E and F). Cosmetic improvement following surgery was favorable (Fig. 3G and $\mathrm{H}$ ).

\section{Case 3}

A 26-year-old man originally presented with headaches, blurry vision, and declining balance. A workup at an outside facility in Kabul led to MRI of the brain (Fig. 4A), which demonstrated a large neoplasm with extensive involvement of the right lateral and third ventricles. Additional superficial extension from the right lateral ventricle into the overlying frontal lobe was also present, as was inferior extension through the floor of the third ventricle into the prepontine cistern. The patient had been sent from Kabul to Peshawar, Pakistan-but for unknown reasons, treatment was not rendered. His mental status continued to decline, and his family brought him to Bagram. On examination, obtundation and slight $(4+/ 5)$ left-sided weakness were present. Repeat CT scan demonstrated interval enlargement of the aforementioned lesion with progression of associated hydrocephalus (Fig. 4B). As the surgery was expected to last beyond 4 hours, a 2 -stage procedure was planned per the request of hospital leadership. In the OR, the patient was placed supine, with his head in Mayfield pin fixation. A bicoronal incision was used to provide access for a right frontal craniotomy. A corticotomy was designed around the site of tumor infiltration into the fron- 

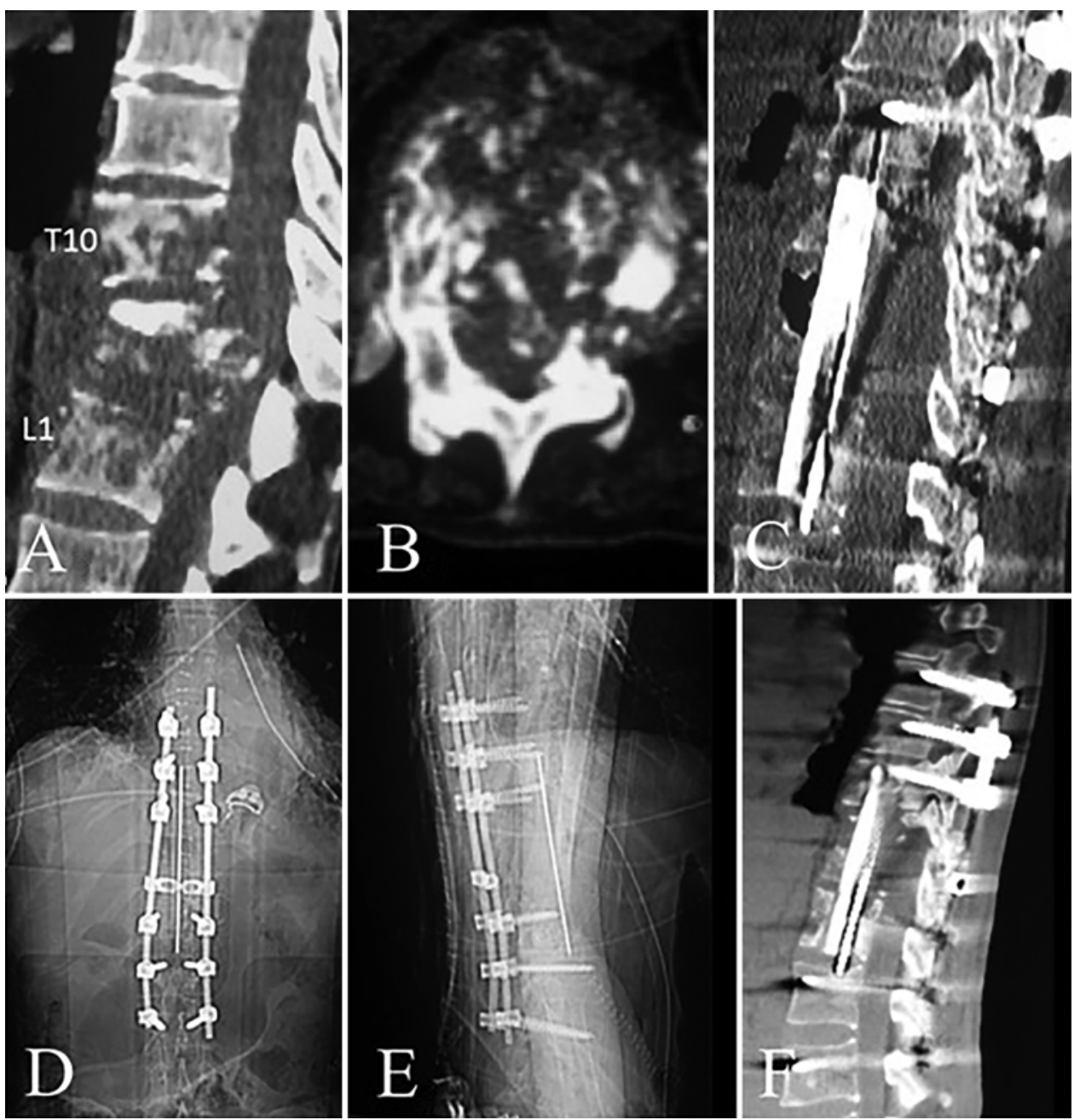

FIG. 5. A: Preoperative sagittal CT image demonstrating moth-eaten appearance of $T 10, T 11, T 12$, and $L 1$ with ventral impingement of the thoracic cord. B: Preoperative axial CT image confirming severe central canal stenosis at the approximate level of T11-12. C: Postoperative sagittal CT image demonstrating full ventral decompression and anterior column reconstruction with fibular autograft. D and E: Postoperative anteroposterior $(\mathrm{D})$ and lateral $(\mathrm{E})$ plain radiographs demonstrating appropriate restoration of alignment after T10-L1 corpectomy, strut graft placement, and T8-L3 posterior fusion. F: Follow-up CT image obtained approximately 5 months after surgery demonstrating early evidence of bridging bone posteriorly with durable correction of deformity.

tal lobe. Right frontal tumor was removed, facilitating a transcortical trajectory to the ventricular system. The ventricular system was entered and the component of tumor in the right lateral ventricle was removed. An external ventricular drain (EVD) was placed prior to closure. In stage 2 , transchoroidal opening of the taenia fornicis was used to further enlarge the opening from right lateral ventricle into the third ventricle. The tumor was seen to originate from the ipsilateral wall of the hypothalamus. The tumor was disconnected here and debulked prior to dissection and removal from the third ventricle and interpeduncular cistern. The EVD was removed in the early postoperative period. The postoperative MRI was consistent with grosstotal resection (Fig. 4C). The final diagnosis, based on pathological examination, was grade II astrocytoma. The patient's headaches, obtundation, and left-sided weakness resolved completely following surgery.

\section{Case 4}

A 35-year-old woman presented with back pain, severe bilateral lower-extremity weakness, and inability to ambulate for 2 months. On examination, she was cachectic and required use of a wheelchair. Her strength was diffusely weak in her bilateral lower extremities (3/5), where marked hyperreflexia was also present. A CT scan of the thoracolumbar spine revealed a moth-eaten appearance of the T10, T11, T12, and L1 vertebral bodies (Fig. 5A and B). There was focal collapse of T11 and T12 with approximately $75 \%$ canal compromise and $20^{\circ}$ of kyphosis while recumbent that worsened when upright. In the setting of severe/progressive paraparesis and spinal deformity secondary to suspected Pott's disease (tuberculous spondylitis), surgery was recommended.

A detailed surgical description of this case has been previously reported. ${ }^{4}$ The first stage of the surgery involved a 4-level (T10-L1) corpectomy with anterior column recon- 
struction using fibular autograft. In the OR, the patient was intubated with a dual-lumen endotracheal tube and placed in the right lateral decubitus position. The initial exposure was provided by cardiothoracic surgery. A thoracoabdominal incision was made over the 10th rib, which was exposed and removed. The thoracic cavity was entered, and the left lung was selectively deflated. The diaphragm was taken down. Inferior to the level of the diaphragm, the peritoneum was retracted anteriorly-exposing the upper lumbar vertebral column. Selected segmental arterial vessels were ligated for hemostasis and a flap of parietal pleura was turned to provide access to the spinal column. The inferior portion of T9, T10, T11, T12, and L1, and the rostral most portion of L2 were exposed. The wall of the thoracolumbar abscess was lanced, and specimens were collected for culture. The left pedicles of T11 and T12 were removed, allowing for excellent visualization of the impinged, anterior thecal sac. The diseased vertebral bodies of T10, T11, T12, and L1 were removed in a piecemeal manner to provide a working space for safe spinal cord decompression. In light of the patient's poor bone quality, the decision was made to preserve the superior endplate of T10 and the inferior endplate of L1, which were noted to be structurally sound. A curette was used to develop the plane between the thecal sac and the posterior longitudinal ligament. The caseous phlegmon that encased the cord at T11 and T12 was dissected away for the thecal sac and collapsed into the working space that had been created. Full decompression of the thecal sac was achieved. No cerebrospinal fluid leak was encountered. The kyphosis was reduced with 3-point pressure and fibular autograft harvested by the orthopedic surgical team was used to reconstruct the 4-level anterior column defect. An intramedullary Kirschner wire (K-wire) was placed in the fibular autograft to enhance radiographic visualization during placement and prevent dislodgement prior to definitive posterior fusion. A chest tube was placed prior to closure in the standard fashion. The patient was then turned prone for a T8 to L3 posterior instrumented fusion. As full ventral decompression had been achieved, posterior decompression was not performed.

The patient noted immediate improvement in strength of both lower extremities following surgery. Postoperative CT confirmed full ventral decompression (Fig. 5C). Postoperative plain radiographs confirmed adequate anterior column reconstruction and restoration of normal alignment (Fig. 5D and E). At the follow-up visit approximately 5 months after surgery, she was noted to have recovered full strength in both lower extremities and was freely ambulating without difficulty. A CT scan performed at that time demonstrated early evidence of bridging bone posteriorly with durable correction of deformity (Fig. 5F).

\section{Case 5}

A 63-year-old professor from Kabul presented with a 1-year history of progressive headaches and visual deterioration. On examination, dense bitemporal hemianopsia was present. CT of the head with contrast demonstrated a large, enhancing sellar neoplasm resulting in severe impingement of the optic apparatus and retrochiasmal extension with mild associated hydrocephalus (Fig. 6A and B). A small amount of tumor extension posterior to the dor-
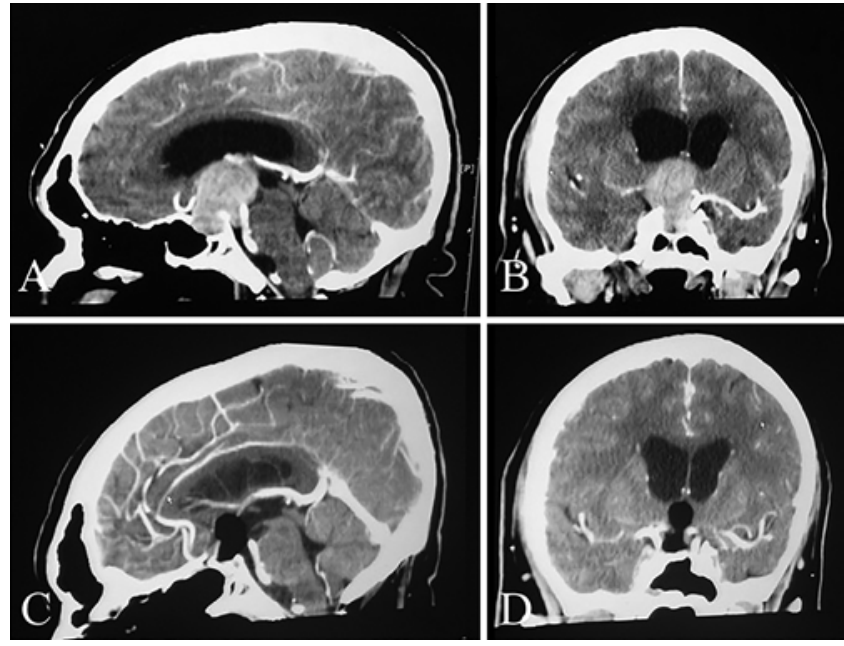

FIG. 6. A and B: Preoperative sagittal (A) and coronal (B) postcontrast CT images demonstrating a large, enhancing sellar neoplasm resulting in severe impingement of the optic apparatus and retrochiasmal extension with mild associated hydrocephalus. C and D: Sagittal (C) and coronal (D) postcontrast CT images obtained in the early postoperative period demonstrating gross-total resection of the lesion, with air in the resection cavity and interval improvement in the ventriculomegaly that was present prior to surgery.

sum sellae was also present. A routine endocrinologic panel supported the diagnosis of nonfunctioning pituitary adenoma. In the setting of visual deterioration, an endoscopic endonasal approach for resection was recommended.

In the OR, initial exposure was provided by an otorhinolaryngologist. Briefly, the middle turbinates were lateralized and a nasoseptal flap was harvested prior to posterior septectomy. Partial ethmoidectomies and a wide sphenoidotomy were performed. At this point, the otorhinolaryngologist "drove" the scope and the remaining exposure and tumor resection was performed by the neurosurgeon (J.A.F.). The floor of the sella and the tuberculum sellae were removed. The lateral margin of bony removal spanned from cavernous sinus to cavernous sinus. The dura was opened widely in a cruciate manner. The tumor was immediately visible. Central debulking was performed prior to extracapsular resection. Following tumor resection, hemostasis was obtained with Gelfoam, and the defect was covered with a nasoseptal flap, which was buttressed with Surgicel at the margins and a Foley balloon catheter.

Postoperatively, the patient noted immediate improvement in his vision. A CT scan with contrast performed in the early postoperative period demonstrated air in the resection cavity with no radiological evidence of residual tumor (Fig. 6C and D). The patient was discharged home after resolution of transient diabetes insipidus. In subsequent follow-up visits, he reported marked improvement in the headaches he suffered from prior to surgery in addition to normalization of vision. On bedside postoperative visual field examination, no residual visual field deficits could be detected.

Case 6

A 25-year-old right-handed man presented with head- 

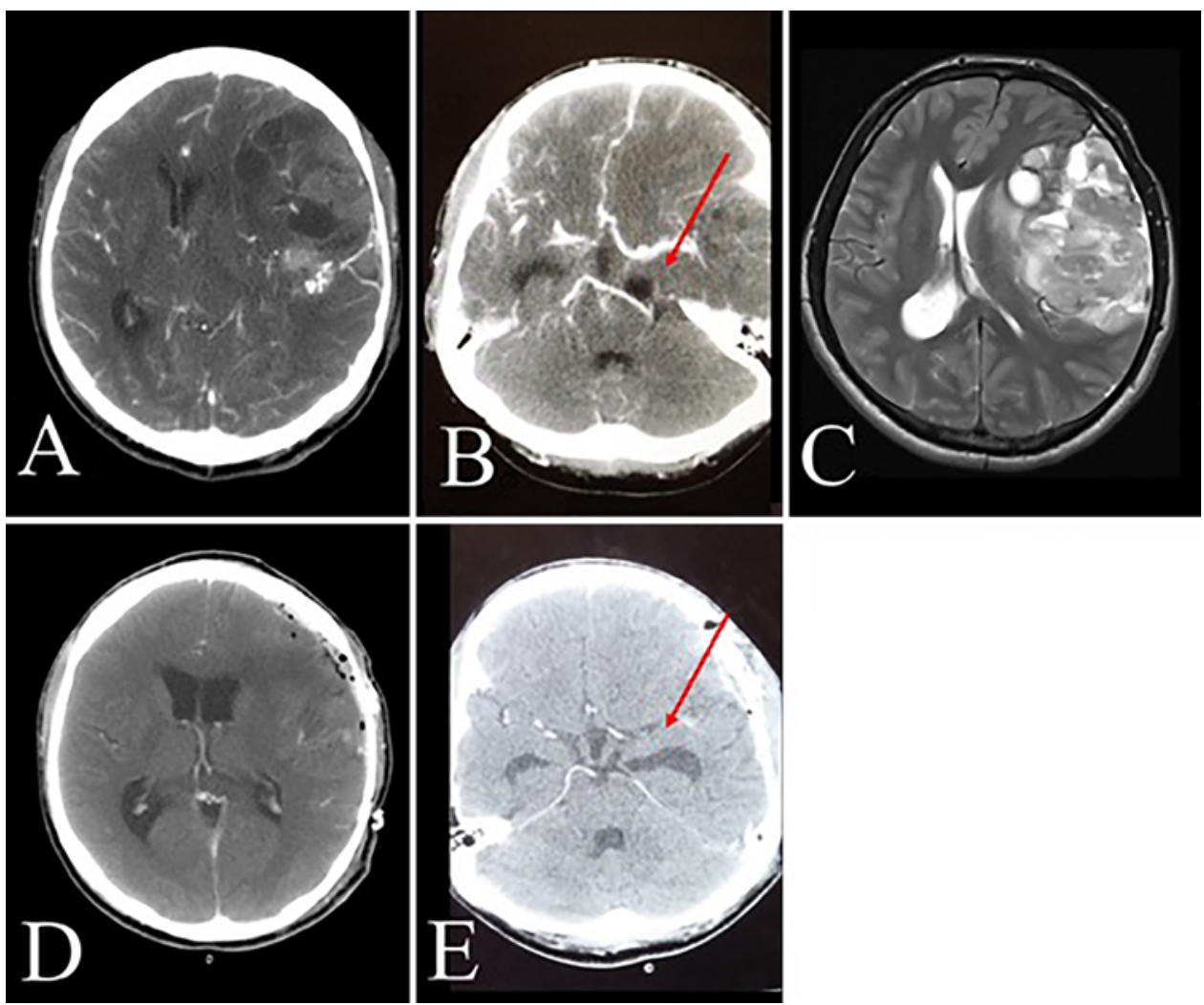

FIG. 7. A and B: Preoperative axial postcontrast CT images demonstrating a large neoplasm on the left exerting mass effect on the ipsilateral frontal and temporal lobes with midline shift. The red arrow indicates radiographic evidence of uncal herniation present prior to surgery. C: T2-weighted MR image demonstrating a heterogeneous neoplasm showing T2 signal hyperintensity. D and E: Postoperative CT images demonstrating effective normalization of midline shift (D) and radiographic uncal herniation (E, red arrow) following resection. No radiographic evidence of residual tumor was present on postoperative imaging.

aches and mild expressive aphasia. A CT scan demonstrated a large intra-axial enhancing neoplasm exerting mass effect on the left frontal and temporal lobes with radiological evidence of uncal herniation (Fig. 7A and B). The middle cerebral artery (MCA) was noted to trifurcate on the inferomedial surface of the tumor. The $\mathrm{M}_{2}$ daughter vessels were intimately associated with the tumor margin. T2-weighted preoperative MRI helped to further characterize the neoplasm (Fig. 7C). Surgery was recommended. In the OR, a curvilinear incision extending from the $\mathrm{zy}$ goma was utilized to turn a left pterional craniotomy. The tumor could be immediately visualized following opening of the dura and was internally decompressed prior to dissection of the tumor margin. The $\mathrm{M}_{1}$ was identified early; great care was taken in preservation of MCA daughter branches during resection. Following completion of the resection, the MCA candelabra could be visualized on the deep and posterior surfaces of the resection cavity. The patient's mild aphasia initially remained stable after surgery; he otherwise remained neurologically intact. No radiographic evidence of residual tumor was present on CT images obtained in the early postoperative period (Fig. 7D and $\mathrm{E})$. The tumor pathology was consistent with grade III oligodendroglioma, positive for loss of heterozygosity of 1p19q. The patient's mild aphasia was noted to have resolved completely 1 month following surgery. He was counseled at this time regarding the benefits of chemotherapy; additional follow-up was arranged with a private hospital in Kabul offering this service.

\section{Discussion}

The Craig Joint Theater Hospital (CJTH) opened at Bagram AFB in 2007 and has remained the largest military medical facility during Operation Enduring Freedom and Operation Freedom's Sentinel since this time. ${ }^{2}$ Expanded opportunities for delivery of humanitarian care (HC) at CJTH became possible in 2010 with opening of the Korean Hospital by South Korea's Provincial Reconstruction Team. ${ }^{5}$ Local Afghans seeking free medical care could gain entry to Bagram AFB via a direct gate to the Korean Hospital. Once inside, they were evaluated by Korean primary care physicians, who served as the gateway for subspecialty referrals. CJTH leadership supported the humanitarian pursuits of American military subspecialty providers, who ran clinics at the Korean facility and performed surgery, when indicated, at CJTH. All surgical cases at CJTH required approval by a weekly multidisciplinary panel to ensure that the proposed care was consistent with guidelines set forth by hospital leadership as well as core ethical principles. ${ }^{11}$ Successful HC at CJTH depended on the efforts of numerous ancillary providers, 
including, but not limited to, anesthesiologists, intensivists, medical internists, and nursing personnel. Closure of the Korean Hospital following completion of the South Korean mission in 2015 eliminated the principal mechanism for patient entry and evaluation at Bagram-resulting in effective termination in elective $\mathrm{HC}$ for local Afghan civilians. Any attempt to restore elective $\mathrm{HC}$ at $\mathrm{CJTH}$ would necessitate re-establishing this gateway for patient entry and evaluation, which undoubtedly would require considerable additional investment on the part of the US military.

Arguments for and against delivery of elective $\mathrm{HC}$ in the current deployed setting of Operation Freedom's Sentinel are complex. Detractors of the HC effort reference the additional costs and security concerns for the US military and cite the unintended financial repercussions delivery of $\mathrm{HC}$ can have on local medical establishments. In general, they feel that the medical effort is unrelated to the overall mission at hand. Supporters of the HC effort commonly reference the considerable medical benefits provided to local Afghan civilians - the vast majority of whom would otherwise be unable to afford care in the fee-for-service model that predominates in the region. They also cite the additional preparation that is afforded to US surgical teams during the sometimes-lengthy intervals between care provided for treatment of battlefield injuries. These proponents feel that delivery of elective $\mathrm{HC}$ is in line with the counterinsurgency platform originally advocated by General Petraeus, in which humanitarian efforts can help "win hearts and minds" and serve as a "force multiplier." 6,8

To the author's knowledge, this paper represents the first consecutive series detailing elective neurosurgical HC provided in a deployed setting. Following a measured reflection of this experience, there are certain points that deserve additional mention. To begin, in the author's opinion, the routine delivery of elective HC undoubtedly strengthened the ability of the neurosurgical team to deliver quality neurosurgical care to critically injured US personnel. During approximately 6 months at Bagram, the CJTH neurosurgical team operated on two active-duty US personnel. In contrast, 49 neurosurgical procedures were performed during delivery of $\mathrm{HC}$. These procedures helped maintain the proficiency of the neurosurgical team in between care of battlefield injuries. Whenever possible, the neurosurgical team at CJTH sought involvement of an Afghan military neurosurgeon during delivery of care. This served to both strengthen the surgical experience of the Afghan military neurosurgeon and provide an additional long-term point of contact in the community for patients undergoing neurosurgical procedures through the $\mathrm{HC}$ effort. As a final note, the provision of $\mathrm{HC}$ strengthened the bond between the US/Afghan military communities and local civilian population. Without question, the relationships forged between the nurses and providers delivering care and patients receiving care created lasting impressions on all involved parties.

\section{Conclusions}

Elective NHC can be safely and effectively implemented in the deployed setting. Benefits of a military strategy that supports $\mathrm{HC}$ include strengthening of the bond between the US/Afghan military communities and the local civilian population as well as maintaining the skills of the neurosurgical team during the sometimes-lengthy intervals between emergent neurosurgical care provided for treatment of battlefield injuries.

\section{References}

1. Causey M, Rush RM Jr, Kjorstad RJ, Sebesta JA: Factors influencing humanitarian care and the treatment of local patients within the deployed military medical system: casualty referral limitations. Am J Surg 203:574-577, 2012

2. Doscher TJ: Bagram gets bigger, better hospital. Stars and Stripes. March 7, 2007 (https://www.stripes.com/news/ bagram-gets-bigger-better-hospital-1.61183) [Accessed October 3, 2018]

3. Edwards MJ, White CE, Remick KN, Edwards KD, Gross KR: Army general surgery's crisis of conscience. J Am Coll Surg 226:1190-1194, 2018

4. Forbes JA, Williams J, Awad AJ, Tiger M, Anderson B, Finnan R: Use of intramedullary K-wire to enhance fibular graft placement in 4-level thoracolumbar corpectomy for Pott disease: case report and review of literature. World Neurosurg 89:404-411, 2016

5. Hyon-hee S: Korean troops bid adieu to Afghanistan. Korea Herald. June 24, 2014 (http://www.koreaherald.com/view. php?ud=20140624000703) [Accessed October 3, 2018]

6. Lischer SK: Military intervention and the humanitarian "force multiplier". Glob Gov 13:99-118, 2007

7. Ragel BT, Klimo P Jr, Kowalski RJ, McCafferty RR, Liu JM, Taggard DA, et al: Neurosurgery in Afghanistan during "Operation Enduring Freedom": a 24-month experience. Neurosurg Focus 28(5):E8, 2010

8. Rosen A: The legacy of David Petraeus and the future of American war. The Atlantic. November 16, 2012 (https:// www.theatlantic.com/international/archive/2012/11/ the-legacy-of-david-petraeus-and-the-future-of-americanwar/265301/) [Accessed October 3, 2018]

9. Sternberg S: Deployments sap surgeons' skills. US News \& World Report. April 19, 2018 (https://www.usnews. com/news/health-care-news/articles/2018-04-19/longdeployments-sap-military-surgeons-skills-proficiency) [Accessed October 3, 2018]

10. Sternberg S, Huth L: Safety in numbers: low volumes at military hospitals imperil patients. US News \& World Report. April 19, 2018 (https://www.usnews.com/news/nationalnews/articles/2018-04-19/patient-shortage-erodes-militarysurgeons-skills-preparedness-for-war) [Accessed October 3, 2018]

11. Welling DR, Ryan JM, Burris DG, Rich NM: Seven sins of humanitarian medicine. World J Surg 34:466-470, 2010

\section{Disclosures}

The author reports no conflict of interest concerning the materials or methods used in this study or the findings specified in this paper.

\section{Correspondence}

Jonathan A. Forbes: University of Cincinnati College of Medicine, Cincinnati, OH. forbesjh@ucmail.uc.edu. 